\title{
Effets d'un pompage à gros débit sur le peuplement des Crustacés d'un aquifère karstique
}

\author{
R. Rouch1 \\ A. Pitzalis 1 \\ A. Descouens 1
}

Mots clés : eaux souterraines karstiques, crustacés stygobies, pompage d'essai.

Un pompage à gros débit dans le gouffre de la Peyrère, regard sur le karst noyé du système karstique du Baget (Ariège, France), a provoqué une dérive importante des microcrustacés stygobies et, notamment, des Harpacticides de ce secteur. Le site du pompage a été partiellement dépeuplé et, un an plus tard, ne semblait pas avoir retrouvé son équilibre antérieur. Ce pompage a également perturbé le peuplement des Harpacticides du drainage en aval du système sans, toutefois, remettre en cause le peuplement global de ce groupe dans le système.

\section{Effects of a high discharge pumping test on the crustacean assemblage of a karstic aquifer}

Keywords : karstic groundwater, stygobitic crustaceans, high discharge pumping test.

A high discharge pumping test performed in a sink-hole connected with the saturated zone of the Baget karstic system (Ariège, France) caused an increased drift of the stygobitic micro-crustaceans, mainly the harpacticoids, of this site. Therefore the pumping site was partially depopulated and, one year later, had not recovered its previous state. Moreover, this pumping test also disturbed the harpacticoid population of the drainage in the down-stream part of the system. Nevertheless, the total harpacticoid population of the system seems to be unaffected by this experience.

Le système karstique du Baget-(Ariège, France) a fait l'objet de nombreuses études tant sur les plans hydrogéologique, géochimique, climatologique qu'hydrobiologique (voir notamment Mangin 1974, 1975, Bakalowicz 1979, Andrieux 1972, Rouch 1974, 1980, 1982). Sa structure et son fonctionnement étant connus, il se prête particulièrement bien à une approche expérimentale des problèmes posés par l'hydrogéologie karstique.

C'est la raison pour laquelle, en octobre 1991, un pompage à gros débit a été réalisé dans un regard sur le karst noyé de ce système, le gouffre de la Peyrère. Cette expérience avait pour but de provoquer un important rabattement de la surface piézométrique dans le gouffre et d'en examiner les conséquences sur le comportement de l'aquifère 2 .

1. Laboratoire souterrain du CNRS, Moulis, 09200 SaintGirons, France.

2. Cette expérience a été menée conjointement par le CNRS et le BRGM.
Dans le cadre de cette opération, plusieurs séries de filtrages des eaux d'exhaure ont été réalisées afin de tenter d'évaluer l'impact d'un tel pompage sur le peuplement des Crustacés du gouffre de la Peyrère d'une part et son éventuelle incidence sur le peuplement du système d'autre part.

\section{Le système karstique du Baget}

\subsection{Caractéristiques générales}

Le bassin d'alimentation de ce système $\left(13,25 \mathrm{~km}^{2}\right)$ s'étend, d'ouest en est, sur un peu plus de $10 \mathrm{~km}$ de long et $1 \mathrm{~km}$ de large environ (Fig. 1). Son altitude moyenne est de $923 \mathrm{~m}$. Les précipitations y sont de l'ordre de $1800 \mathrm{~mm} / \mathrm{an}$. Le débit moyen du système s'établit à $0,523 \mathrm{~m}^{3} / \mathrm{s}$, les débits minimal et maximal observés étant de $0.050 \mathrm{~m}^{3} / \mathrm{s}$ et $10,9 \mathrm{~m}^{3} / \mathrm{s}$.

Les terrains non calcaires représentent près de $33 \%$ de sa superficie (Mangin 1.974, 1975). Vers l'aval, le système est profondément entaillé par la 
vallée de Lachein qui suit l'axe longitudinal du bassin. Habituellement sèche, cette vallée devient le siège d'écoulements temporaires importants lors des forts épisodes pluvieü sous la double influence du ruissellement de surface sur les terrains imperméables et des apports par les exutoires de trop-plein. En effet, outre l'exutoire pérenne du système, Las Hountas (498 m), il existe plusieurs émergences de trop-pleins de l'aval vers l'amont : le Moulo de Jaur tout d'abord, puis l'ensemble des griffons du secteur de La Hillère (dont HR 121, HR 25, HR 241). Deux gouffres, Sainte-Catherine au nord, la Peyrère au sud atteignent le karst noyé.

\subsection{La structure du kàrst noyé}

Selon le modèle général proposé par Mangin (1974, 1975), le kart noyé est composé d'un drainage (transmissif mais peu capacitif) et de systèmes annexes au drainage (capacitifs mais peu transmissifs) isolés les uns les autres mais tous en relation avec le drainage. C'est ainsi que, dans la partie en aval du Baget, deux systèmes annexes au drainage ont été reconnus en rive droite de la vallée du Lachein : celui de la Hillère (exutoires HR25 et HR241) et celui de la Peyrère (gouffre de la Peyrère). Les exutoires de trop-plein du Moulo de Jaur et de HR121 sont, au contraire, en liaison avec le drainage (Fig. 1).

\section{Le peuplement des Crustacés du système karstique du Baget}

Le système karstique du Baget possède une riche faune de Crustacés mise en évidence par l'étude de la dérive au niveau des exutoires. 50 espèces de Copépodes, Ostracodes, Syncarides, Isopodes et Amphipodes y ont été recensées (Tableau 1). Les Copépodes représentent le groupe le plus abondant et le plus diversifié de cette dérive avec, notamment, les Harpacticides (21 espèces dont 8 stygobies).

\subsection{Les modalités de la dérive des Harpacticides}

Au moment des crues, les Harpacticides font l'objet d'une dérive de type catastrophique (Rouch $1970,1972,1974)$ dont l'ampleur n'est mathématiquement pas corrélée au débit (Rouch 1982). Le tableau 2 rassemble les valeurs du taux de dérive horaire moyen (D) et de la densité de dérive moyenne (d) concernant le premier jour de crue de 30 crues étudiées à Las Hountas entre 1971 et 1982. Ces taux et densités de dérive ne sont corrélés ni avec l'amplitude (QB1 maximum) de la crue ( $\mathrm{r}=0,104$ pour $\mathrm{rc}=0,349$ au seuil de $5 \%$ avec $30 \mathrm{dl}$ ) ni avec le débit moyen de Las Hountas (Q) pendant le filtrage $(r=0,184$ pour $r c=0,349$ au seuil de $5 \%$ avec $30 \mathrm{dl}$ ). Les taux de dérive horaire moyens sont compris entre 1500 et 12500 individus, les densités

Tableau 1. Liste des espèces de Crustacés du système karstique du Baget.

Table 1. List of the crustacean species from the Baget karstic system.

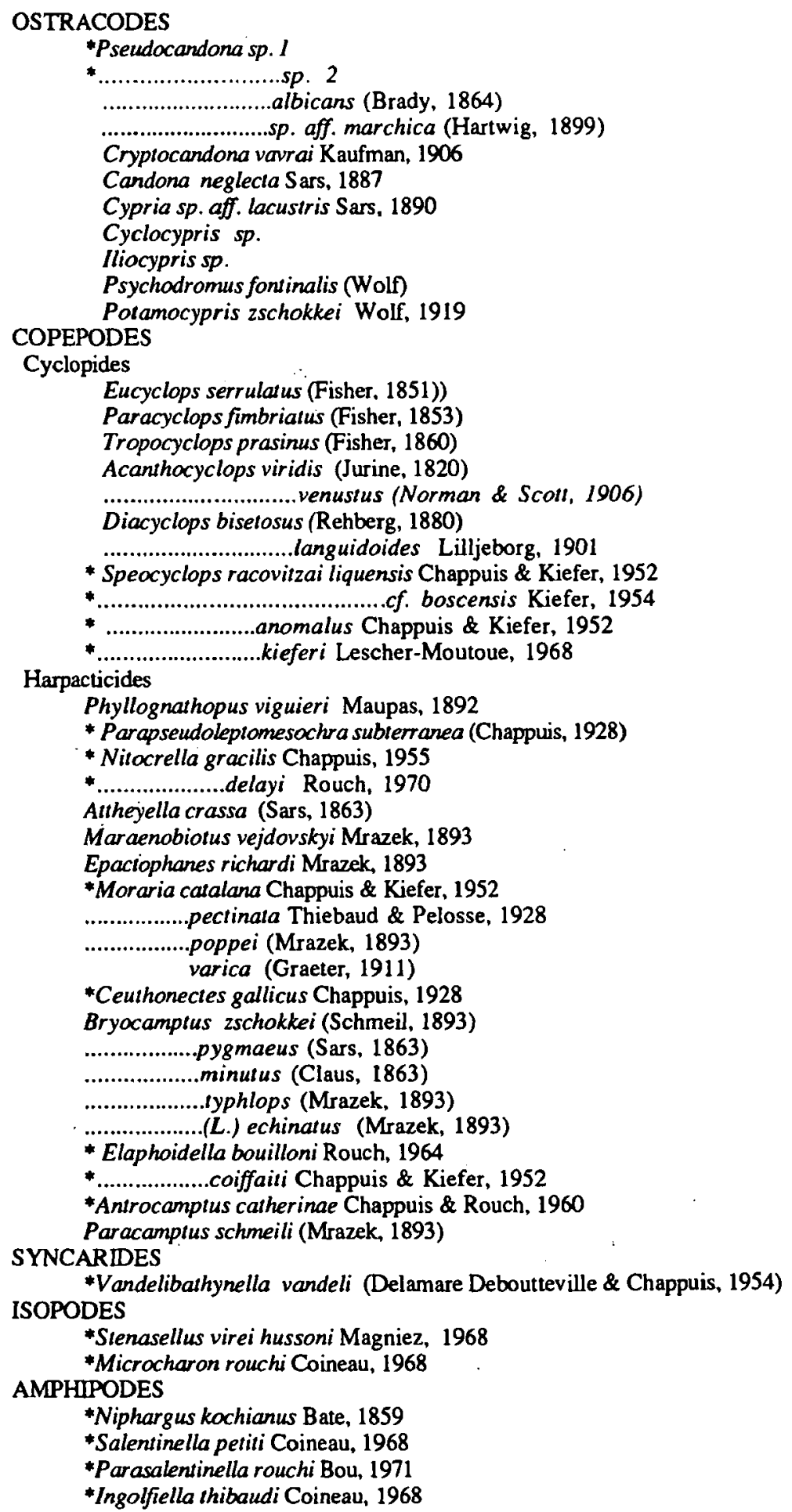


entre 0,5 et $4 \mathrm{i} / \mathrm{m}^{3}$. Les formes stygobies représentent $52 \%$ du transit observé.

\subsection{La structure du peuplement des Harpacticides hypogés}

Les différents exutoires du système du Baget sont caractérisés par leur identité quant à la composition taxonomique de la dérive dont ils font l'objet (Rouch \& Bonnet 1976).
Toutefois, les réponses des exutoires n'en sont pas moins très différentes si l'on tient compte des abondances relatives des espèces. Lors de 7 crues, survenues entre janvier 1973 et novembre 1974, des filtrages ont été réalisés de façon synchrone à Las Hountas, le Moulo de Jaur, HR 121 et HR 241 (voir Fig. 1). Au total, 26 échantillons de dérive ont été prélevés. Ces échantillons ont fait l'objet d'une analyse factorielle des correspondances (Rouch \&
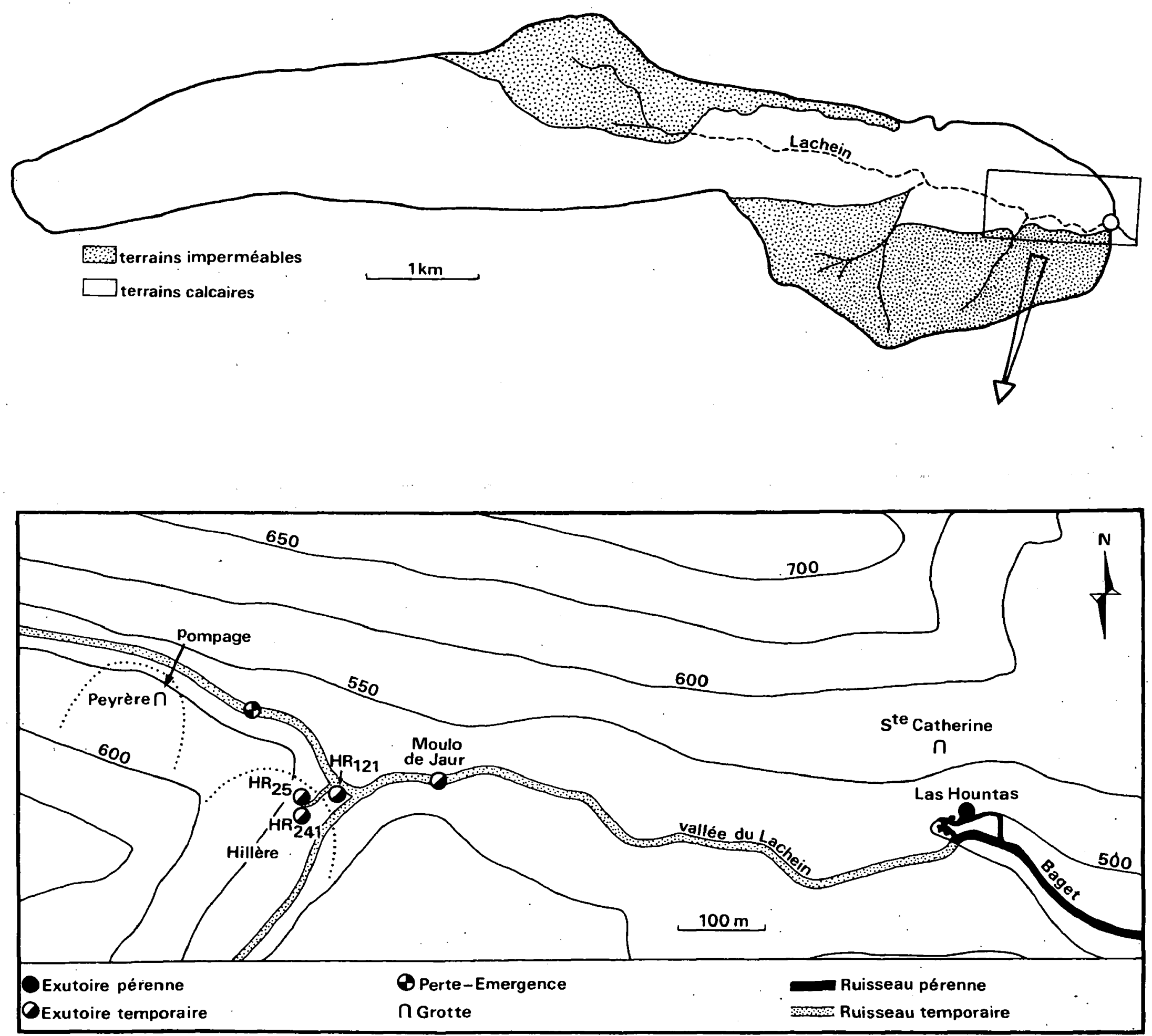

Fig. 1. Carte du système karstique du Baget.

Fig. 1. Map of the Baget karstic system. 
Tableau 2. Valeurs moyennes de la dérive des Harpacticides à Las Hountas, le premier jour de crue, lors de $30 \mathrm{crues}$. QB1 max = débit maximum du système. $Q$ Hountas = débit moyen de Las Hountas pendant la période de filtrage. $\mathrm{D} / \mathrm{h}:$ nombre moyen d'individus entraînés pendant une heure. $d / \mathrm{m}^{3}$ : nombre moyen d'individus par $\mathrm{m}^{3}$.

Table 2. Mean values of the harpacticoid drift at the spring Las Hountas during the first day of 30 floods. QB1 max $=$ maximum discharge of the system. QHountas = average discharge of the Las Hountas spring during the sampling period. D/h : average drift rate. $d / m^{3}$ : average drift density.

\begin{tabular}{|c|c|c|c|c|c|c|}
\hline \multicolumn{2}{|c|}{ Crues } & \multirow{2}{*}{$\begin{array}{c}\text { durée } \\
\text { filtr. } \\
\text { (h) }\end{array}$} & \multirow{2}{*}{$\begin{array}{l}\text { QB1 } \\
\max \\
\left(\mathrm{m}^{3}\right)\end{array}$} & \multirow{2}{*}{$\begin{array}{c}\text { Q Hountas } \\
\text { (filtrage) } \\
\text { (m3) }\end{array}$} & \multirow{2}{*}{$\mathrm{D} / \mathrm{h}$} & \multirow{2}{*}{$\mathrm{d} / \mathrm{m}^{3}$} \\
\hline $\mathrm{II}^{0}$ & date & & & & & \\
\hline$\overline{1}$ & 6.1 .71 & 7 & 1,140 & 0,592 & 8441 & 4,01 \\
\hline 2 & 20.1 .71 & 9 & 1,060 & 0,587 & 7378 & 2,72 \\
\hline 3 & 17.2 .71 & 9 & 3,950 & 0,785 & 4774 & 1,76 \\
\hline 4 & 22.11 .71 & 7 & 3,150 & 0,886 & 3599 & 1,12 \\
\hline 5 & 12.1.72 & 12 & 1,240 & 0,599 & 1956 & 0,91 \\
\hline 6 & 18.1.72 & 9 & 1,730 & 0,663 & 2259 & 0,95 \\
\hline 7 & 12.4 .72 & 9 & 4,650 & 1,155 & 3468 & 0,84 \\
\hline 8 & 1.12 .72 & 9 & 0,640 & 0,556 & 2199 & 1,10 \\
\hline 9 & 29.1 .73 & 5 & 5,150 & 0,931 & 3811 & 1,13 \\
\hline 10 & 24.2.73 & 8 & 8,000 & 1,006 & 2645 & 0,71 \\
\hline 11 & 11.5 .73 & 9 & 1,780 & 0,705 & 1694 & 0,67 \\
\hline 12 & 30.11 .73 & 9 & 1,415 & 0,608 & 3981 & 1,82 \\
\hline 13 & 5.2 .74 & 7 & 3,950 & 0,817 & 3215 & 1,08 \\
\hline 14 & 17.9 .74 & 8 & 4,500 & 1,112 & 12432 & 3,11 \\
\hline 15 & 27.11 .74 & 8 & 4,400 & 1,076 & 2488 & 0,63 \\
\hline 16 & 17.11 .75 & 5 & 1,040 & 0,589 & 3588 & 1,69 \\
\hline 17 & 14.2.76 & 8 & 2,740 & 0,767 & 2226 & 0,81 \\
\hline 18 & 18.10 .76 & 6 & 3,100 & 0,785 & 2836 & 1,01 \\
\hline 19 & 3.12 .76 & 8 & 4,800 & 1,103 & 4319 & 1,08 \\
\hline 20 & 12.4.77 & 5 & 2,390 & 0,821 & 3497 & 1,18 \\
\hline 21 & 4.5 .77 & 8 & 2,830 & 0,808 & 2652 & 0,89 \\
\hline 22 & 10.11 .79 & 8 & 0,740 & 0,548 & 2628 & 1,33 \\
\hline 23 & 24.4 .80 & 8 & 0,850 & 0,532 & 1975 & 1,03 \\
\hline 24 & 31.3 .81 & 8 & 1,795 & 0,699 & 2249 & 0,90 \\
\hline 25 & 17.4 .81 & 8 & 1,370 & 0,627 & 2298 & 1,02 \\
\hline 26 & 24.4 .81 & 8 & 2,155 & 0,706 & 2026 & 0,81 \\
\hline 27 & 28.10 .81 & 8 & 1,140 & 0,595 & 3904 & 1,82 \\
\hline 28 & 2.12 .81 & 8 & 2,515 & 0,730 & 2947 & 1,12 \\
\hline 29 & 14.12 .81 & 8 & 6,080 & 1,371 & 2712 & 0,55 \\
\hline 30 & $27.1: 82$ & 6 & 1,930 & 0,631 & 1541 & 0,68 \\
\hline
\end{tabular}

Bonnet 1977) dans laquelle n'ont été prises en compte que les espèces stygobies dominantes: Parapseudoleptomesochra subterranea, Nitocrella gracilis, Elaphoidella coiffaiti, Ceuthonectes gallicus. La figure obtenue dans le plan factoriel F1-F2 (Fig. 2) montre que les échantillons issus d'un même exutoire ne peuvent être confondus avec ceux provenant des autres émergences (à l'exception d'un chevauchement entre Las Hountas et le Moulo de Jaur). En outre, l'organisation spatiale de ces échantillons se superpose à la structure hydrogéologique du karst noyé. Las Hountas, le Moulo de Jaur et HR 121 se distribuent le long de l'axe 2 de la figure selon leur position respective sur le drainage de l'aval vers l'amont. Ils sont caractérisés par l'abondance relative élevée de $P$. subterranea (60\%). HR 241, émergence de trop-plein du système annexe de $\mathrm{La}$ Hillère, détermine l'axe 1 . A cet endroit, la dérive est dominée par C. gallicus (47 \% au lieu de $24 \%$ dans le drainage).

Ainsi, mois après mois, crues après crues, les réponses des différents exutoires demeurent relativement identiques prouvant ainsi que chaque secteur étudié possède une signature biologique qui lui est propre.

\subsection{Le peuplement des Crustacés du gouffre de La Peyrère}

Ce gouffre abrite une abondante population de l'Isopode Stenasellus virei hussoni. En l'absence d'émergence de trop-plein qui aurait permis d'étudier la dérive issue de ce secteur du Baget, le peuplement des micro-crustacés du gouffre de la Peyrère peut être défini à partir de deux pêches successives qui y ont été réalisées à l'étiage, les 2 et 
9 octobre 1975 (Tableau 3). Les Harpacticides constituent le groupe le mieux représenté $(88,8 \%)$. $99 \%$ d'entre eux appartiennent à des espèces stygobies parmi lesquelles on retrouve les 4 espèces dominantes dans la dérive aux exutoires plus une espèce de Parastenocaris qui elle n'y participe jamais. En se fondant sur l'étude de ce groupe, le gouffre de la Peyrère se singularise des autres secteurs du système en raison de l'abondance relative de $N$. gracilis $(76,1 \%)$. Cette espèce ne dépasse jamais $7 \%$ dans le drainage et n'atteint que $4 \%$ dans le système annexe de la Hillère (HR 241).

Sur la base de ces données, la spécificité du secteur de la Peyrère ne fait donc pas de doute par rapport, d'une part, au drainage (caractérisé par $P$. subterranea) et, d'autre part, au secteur de la Hillère (défini par C. gallicus).

\section{Le pompage dans le gouffre de la Pey- rère : données hydrologiques 1}

L'entrée du gouffre étant située à $560 \mathrm{~m}$ d'altitude et le niveau de la surface piézométrique à $502 \mathrm{~m}$, trois groupes de 3 pompes se relayant les uns les autres ont été nécessaires pour refouler l'eau hors de la cavité. Le premier était immergé à $-24 \mathrm{~m}$ sous le niveau de la surface piézométrique ( $478 \mathrm{~m}$ d'altitude). A partir du rebord du gouffre, l'eau, s'écoulant par gravité, était acheminée par une conduite au-delà de l'exutoire pérenne $(1 \mathrm{~km})$ afin de ne pas réalimenter le système. Un tube coudé, placé à

1. Les données hydrologiques relatives à ce pompage sont extraites du rapport établi par le Laboratoire souterrain et le BRGM.

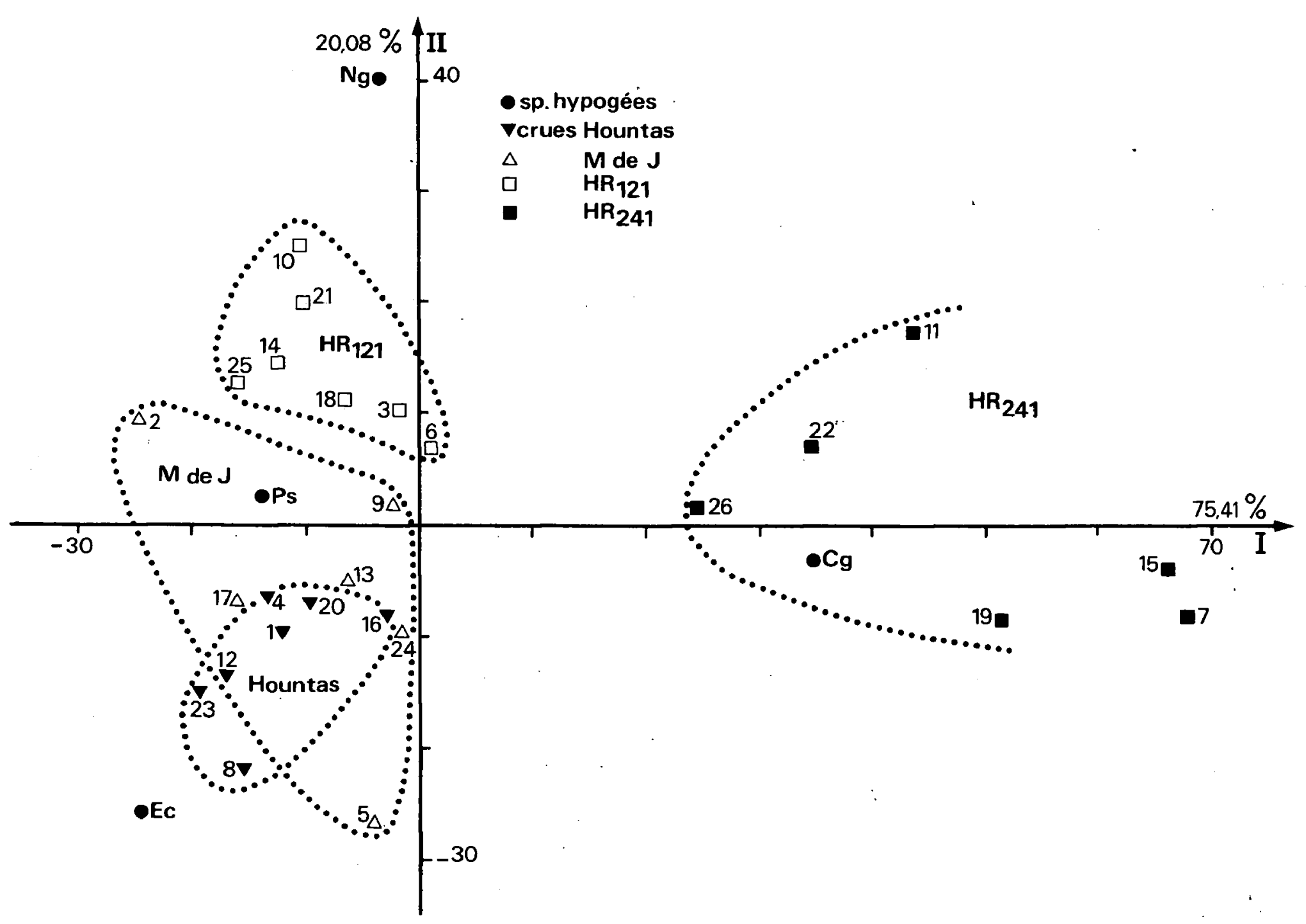

Fig. 2. Analyse factorielle des correspondances sur 26 échantillons de dérive provenant de Las Hountas, le Moulo de Jaur, HR 121 et HR 241 ; seuls les quatre Harpacticides stygobies dominants ont été pris en compte.

Fig. 2. Correspondence analysis on 26 synchronous drift samples collected in four springs of the Baget karstic system (Las. Hountas, Moulo de Jaur, HR 121, HR 241). Only the four dominant stygobiont harpacticoid species have been used. 
Tableau 3. Liste des individus récoltés dans le gouffre de la Peyrère.

Table 3. List of the crustaceans collected in the La Peyrère top window.

\begin{tabular}{|c|c|c|c|c|c|c|c|c|}
\hline \multirow[t]{2}{*}{ Crustacés } & \multicolumn{2}{|c|}{ pêches 1975} & \multicolumn{2}{|c|}{ pompage 1991} & \multicolumn{2}{|c|}{$\begin{array}{l}\text { prélèvements } \\
\text { in situ } 1991\end{array}$} & \multicolumn{2}{|c|}{ pêches 1992} \\
\hline & nb. i. & $\%$ & ng. i. & $\%$ & nb. i. & $\%$ & nb. i. & $\%$ \\
\hline \multicolumn{9}{|l|}{ Harpacticides } \\
\hline N. gracilis & 1107 & 76,1 & 290 & 30,6 & 46 & 24,5 & 2 & $.1,4$ \\
\hline P. subterranea & 46 & 3,2 & 135 & 14,6 & 18 & 9,6 & 26 & 18,8 \\
\hline C. gallicus & 139 & 9,6 & 502 & 52,9 & 118 & 62,7 & 80 & 58 \\
\hline E. coiffaiti & 9 & 0,6 & 4 & 0,4 & & & & \\
\hline P. mangini & 139 & 9,6 & 4 & 0,4 & & & & \\
\hline T. stygobies & 1440 & 99 & 935 & 98,5 & 182 & 96,8 & 108 & 78,2 \\
\hline B. zschokkei & 6 & 0,4 & 3 & 0,3 & & & 3 & 2,2 \\
\hline B. typhlops & 4 & 0,3 & & & & & 2 & 1,4 \\
\hline B. (L.) echinatus & 4 & 0,3 & 10 & 1,1 & 4 & 2,1 & 15 & 10,9 \\
\hline A. crassa & & & & & & & 10 & 7,2 \\
\hline M. poppei & & & & & 2 & 1,1 & & \\
\hline P. viguieri & & & 1 & 0,1 & & & & \\
\hline T. épigés & 14 & 1 & 14 & $l, 5$ & 6 & 3,2 & 30 & 21,7 \\
\hline T. général & \multicolumn{2}{|c|}{$1454(88,8 \%)$} & \multicolumn{2}{|c|}{$949(83,2 \%)$} & \multicolumn{2}{|c|}{$188(76,7 \%)$} & \multicolumn{2}{|c|}{$138(80,7 \%)$} \\
\hline Cyclopides & \multicolumn{2}{|c|}{$118(7,2 \%)$} & \multicolumn{2}{|c|}{$141(12,4 \%)$} & \multicolumn{2}{|c|}{$48(19,6 \%)$} & \multicolumn{2}{|c|}{$33(19,3 \%)$} \\
\hline $\begin{array}{l}\text { Syncarides } \\
\text { V. vandeli }\end{array}$ & \multicolumn{2}{|c|}{$65(4 \%)$} & \multicolumn{2}{|c|}{$40(3,5 \%)$} & \multicolumn{2}{|c|}{$6(2,4 \%)$} & & \\
\hline Amphipodes & & & \multirow{2}{*}{\multicolumn{2}{|c|}{$2(0,2 \%)$}} & \multirow{4}{*}{\multicolumn{2}{|c|}{$3(1,2 \%)$}} & & \\
\hline N. kochianus & & & & & & & & \\
\hline S. petiti & & & & & & & & \\
\hline I. thibaudi & & & & $\%)$ & & & & \\
\hline Total général & \multicolumn{2}{|c|}{1637} & \multicolumn{2}{|c|}{1140} & \multicolumn{2}{|c|}{245} & \multicolumn{2}{|c|}{171} \\
\hline
\end{tabular}

à l'extrémité distale de cette conduite, permettait de . dériver une partie des écoulements $(2 \mathrm{l} / \mathrm{s})$ vers un filet de $100 \mu \mathrm{m}$ de maille.

Trois essais successifs ont été réalisés entre le 24 et le 28 octobre 1991 (Tableau 4).

Pour l'ensemble de ces trois essais, le volume d'exhaure a représenté $43650 \mathrm{~m}^{3}$ (débit moyen = $225 \mathrm{l} / \mathrm{s}$ ). En l'absence de pompage, l'écoulement naturel du système aurait fourni $28350 \mathrm{~m}^{3}$ (débit moyen de Las Hountas $=141 \mathrm{l} / \mathrm{s}$ ). Un volume de $15300 \mathrm{~m}^{3}$ a donc été prélevé dans la réserve (6 $600 \mathrm{~m}^{3}$ lors du premier pompage, $5700 \mathrm{~m}^{3}$ lors du deuxième, $3000 \mathrm{~m}^{3}$ lors du troisième) ce qui correspond à un débit de surexploitation de $80 \mathrm{l} / \mathrm{s}$.

Cette surexploitation du système a eu pour conséquence plusieurs phénomènes.

La surface piézométrique dans le gouffre de la Peyrère (Fig. 3) a subi trois rabattements successifs importants $(21,5 \mathrm{~m} ; 20,5 \mathrm{~m} ; 12,5 \mathrm{~m})$. Ainsi, à la fin du premier pompage, le niveau de l'eau dans le gouffre était à $-17,5 \mathrm{~m}$ par rapport au niveau de Las Hountas. Dans ces conditions, l'exhaure de l'aquifère a été détourné vers le gouffre. La conséquence la plus spectaculaire a résidé, lors des trois essais, dans l'assèchement progressif de Las Hountas. Celui-ci a débuté dès que le plan d'eau dans le gouffre a atteint $-4 \mathrm{~m}$. En quelques heures, Las Hountas s'est asséchée presque totalement (débit résiduel de $7 \mathrm{l} / \mathrm{s}$ ). Après l'arrêt des pompages, le niveau piézométrique dans le gouffre a retrouvé sa cote initiale $(502 \mathrm{~m})$ en 14,13 et 10 heures. Dans le même temps, les écoulements à Las Hountas se rétablissaient progressivement.

Avant le pompage, le niveau de l'eau dans le système annexe de la Hillère (HR 25 Fig. 1 et 3) était de $504 \mathrm{~m}$, soit 6 mètres au-dessus de celui de Las Hountas. Il était donc plus élevé que celui de 
Tableau 4. Les trois pompages dans le gouffre de la Peyrère : durées, caractéristiques hydrologiques, temps de filtrage.

Table 4. The three pumping tests in the La Peyrère top-window : duration, hydrological characteristics, sampling duration.

\begin{tabular}{|c|c|c|c|}
\hline Pompage & $1^{\text {er }}$ essai : & $2^{e}$ essai & $3^{e}$ essai \\
\hline début & $24.10 .91 \quad 12$ h 30 & $26.10 .9110 \mathrm{~h}$ & 28.10 .9110 h 30 \\
\hline fin & $25.10 .91 \quad 16$ h 30 & $27.10 .91 \quad 4 \mathrm{~h}$ & $28.10 .91 \quad 18$ h 30 \\
\hline durée & $28 \mathrm{~h}$ & $18 \mathrm{~h}$ & $8 \mathrm{~h}$ \\
\hline débit & $221 \mathrm{l} / \mathrm{s}$ & $228 \mathrm{l} / \mathrm{s}$ & $230 \mathrm{l} / \mathrm{s}$ \\
\hline rabattement & $21,5 \mathrm{~m}$ & $20,5 \mathrm{~m}$ & $12,5 \mathrm{~m}$ \\
\hline volume & $22230 \mathrm{~m}^{3}$ & $14760 \mathrm{~m}^{3}$ & $6600 \mathrm{~m}^{3}$ \\
\hline Filtrage & $\begin{array}{c}24.10 .91 \\
(13 \text { h } 45-22 \text { h } 45) \\
25.10 .91 \\
(8 \text { h } 20-16 \text { h } 20)\end{array}$ & $\begin{array}{c}26.10 .91 \\
(10 h-19 h)\end{array}$ & $\begin{array}{c}28.10 .91 \\
(10 \text { h } 30-18 h)\end{array}$ \\
\hline durée & $9 h+8 h$ & $9 \mathrm{~h}$ & 7 h 30 \\
\hline
\end{tabular}

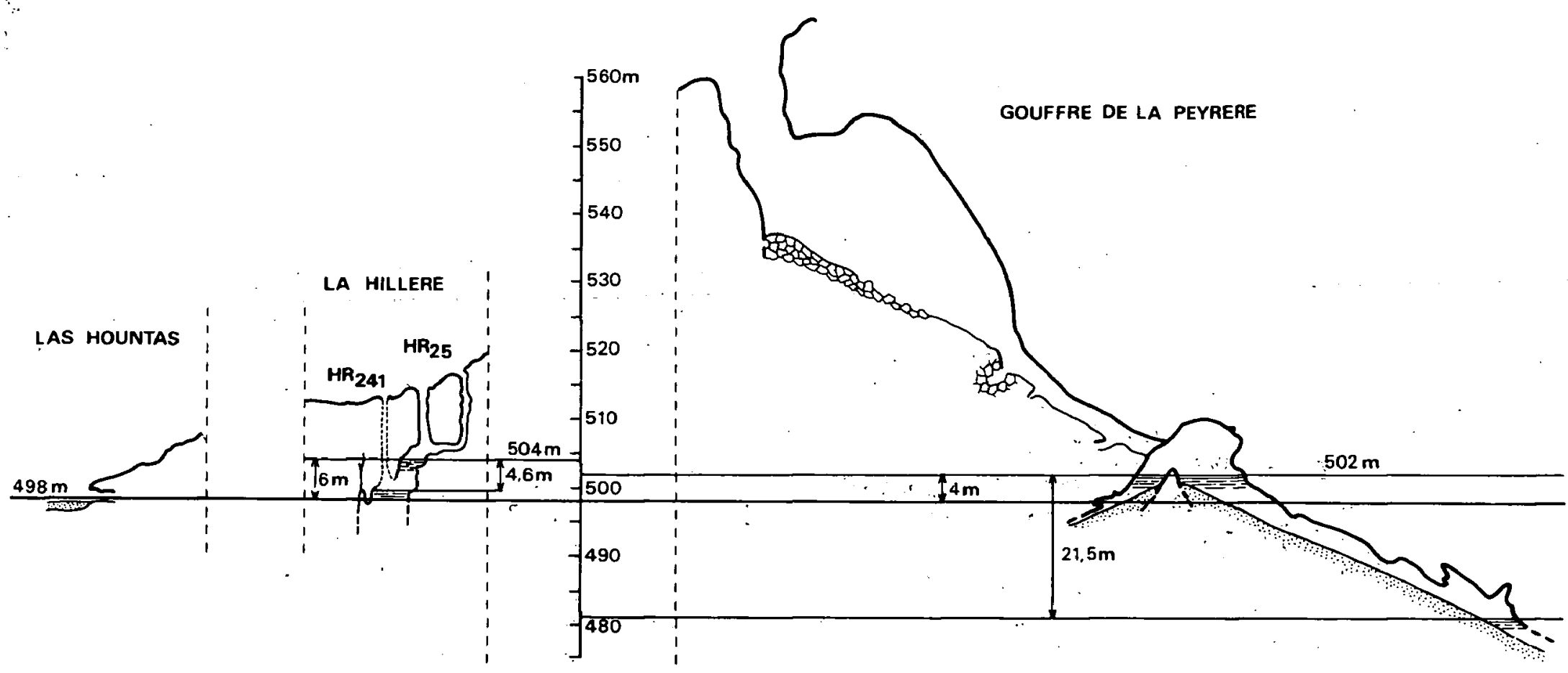

Fig. 3. Cotes de la surface piézométrique, avant le pompage et lors du rabattement maximum, dans le gouffre de la Peyrère et dans le secteur de la Hillère (au niveau des exutoires HR 25 et HR 241). La coupe du gouffre de la Peyrère est due à G. Jauzion de la SMSP.

Fig. 3. Level of the piozemetric surface before the pumping test and during the maximum draw down in the la Peyrere top window and the La Hillère sector (HR 25 and HR 241 springs). The La Peyrère section was produced by G. Jauzion.

la Peyrère (502 m), bien que La Hillère soit en aval du gouffre, ce qui confirme bien l'indépendance des systèmes annexes les uns par rapport aux autres. Au cours des trois essais, la surface piézométrique de La Hillère s'est abaissée de 4,6 m pour se stabiliser à la cote de 499,4 m. Cette stabilisation à $19 \mathrm{~m}$ audessus du niveau le plus bas atteint à la Peyrère, implique que le secteur de la Hillère s'est chaque fois déconnecté du drainage. 


\section{Le pompage dans le gouffre de la Pey- rère : données biologiques}

Ces données concernent d'une part la dérive provoquée par le pompage et, d'autre part, des prélèvements effectués dans le gouffre lors du rabattement de la surface piézométrique (1 ${ }^{\mathrm{er}}$ essai). Seuls les Crustacés ont été pris en compte et, plus particulièrement, les Harpacticides.

\subsection{Image faunistique globale des Crustacés dans la dérive}

Malgré sa présence dans le gouffre, Stenasellus virei hussoni n'est jamais apparu dans la dérive provoquée par le pompage (Tableau 3). Les Harpacticides représentent 83,2 \% des 1140 Crustacés capturés, les Cyclopides $12,4 \%$, les Syncarides 3,5 \% et les Amphiphodes 0,9\%. Globalement, l'image taxonomique fournie par le pompage est proche de celle de la pêche réalisée en 1975. Chez les Harpacticides, le pourcentage des formes stygobies s'établit à $98,5 \%$ pour $99 \%$ en 1975 . Toutefois, bien que les espèces stygobies soient les mêmes que celles récoltées en 1975, leur abondance relative est différente.

\subsection{La dérive des Harpacticides : approche quantitative}

Puisque les écoulements filtrés $(2 \mathrm{l} / \mathrm{s})$ représentaient le 1/100 environ du débit de pompage, les taux de dérive globaux (nombre d'individus entraînés par heure) ont été estimés en multipliant par 100 les taux de dérive partiels fournis par les filtrages horaires. Les densités de dérive (nombre d'individus $/ \mathrm{m}^{3}$ ) ont été calculées directement à partir des données du filtrage. En opérant ainsi, il était implicitement supposé que les animaux entraînés étaient uniformément répartis dans la conduite d'exhaure.

\subsubsection{Premier essai (Fig. 4a)}

Les taux de dérive partiels ont varié entre 10 et $82 \mathrm{i} / \mathrm{h}$ et les densités de dérive entre 1,4 et $11,4 \mathrm{i} / \mathrm{m}^{3}$.

Les taux de dérive globaux, compris entre 1000 et $8200 \mathrm{i} / \mathrm{h}$, permettent d'estimer à $48000 \mathrm{le}$ nombre d'Harpacticides entraînés lors de la première séquence et à 18000 ceux évacués lors de la seconde. En intrapolant les valeurs intermédiaires, la dérive totale des Harpacticides lors de ce premier essai aurait été de l'ordre de 100000 individus.
La chronologie des taux de dérive lors de la première séquence montre que ceux-ci passent par un point bas entre 16 et 18 heures ( 4 heures après le début du pompage) avant de croître de nouveau. Or, l'examen de la composition faunistique des échantillons (Fig. 4) indique qu'ils sont caractérisés, au début, par $N$. gracilis ( $>70 \%$ des Harpacticides capturés). Ensuite, pendant la période de faible dérive, le pourcentage de $N$. gracilis décroît brutalement pour se stabiliser autour de valeurs voisines de $10 \%$. N. gracilis est alors remplacée par C. gallicus. Le lendemain, le signal est moins bien structuré mais $C$. gallicus reste globalement dominant.

\subsubsection{Deuxième essai (Fig. 4b)}

Au cours de la première heure, la dérive dans les écoulements filtrés est importante $(91 \mathrm{i} / \mathrm{h})$ puis elle s'effondre à moins de $10 \mathrm{i} / \mathrm{h}$ pour remonter ensuite à $30 \mathrm{i} / \mathrm{h}$. Les densités de dérive ont varié entre 0,5 et $12,6 \mathrm{i} / \mathrm{m}^{3}$ et les taux de dérive pour l'ensemble des écoulements entre 400 et $9100 \mathrm{i} / \mathrm{h}$.

La dérive totale pour ces 9 heures de pompage peut être estimée à 20000 individus soit $40 \%$ de celle obtenue pour la première séquence du premier pompage.

Pendant les cinq premières heures de ce deuxième essai, $N$. gracilis est l'espèce dominante de la dérive ( $>50 \%$ des individus capturés). Elle est ensuite relayée par $C$. gallicus qui fournit plus de $70 \%$ des Harpacticides récoltés et qui assure un regain de dérive:

\subsubsection{Troisieme essai (Fig. 4c)}

Le nombre d'individus capturés, très faible au début $(<10 \mathrm{i} / \mathrm{h})$, atteint $30 \mathrm{i} / \mathrm{h}$ en fin de pompage. Les densités de dérive ont varié entre 0,1 et $4,1 \mathrm{i} / \mathrm{m}^{3}$ et les taux de dérive globaux entre 100 et $3000 \mathrm{i} / \mathrm{h}$.

Au cours de cet essai, la dérive totale des Harpacticides a été de l'ordre de 9000 individus, soit $20 \%$ seulement de celle enregistrée pendant la première séquence du premier pompage (bien qu'inégaux, les temps de filtrage de ces deux séquences sont comparables).

La composition faunistique de ce signal est beaucoup moins nette que précédemment puisque, au moment où la dérive est la plus importante, les pourcentages de $N$. gracillis et $C$. gallicus sont proches. 
(A)

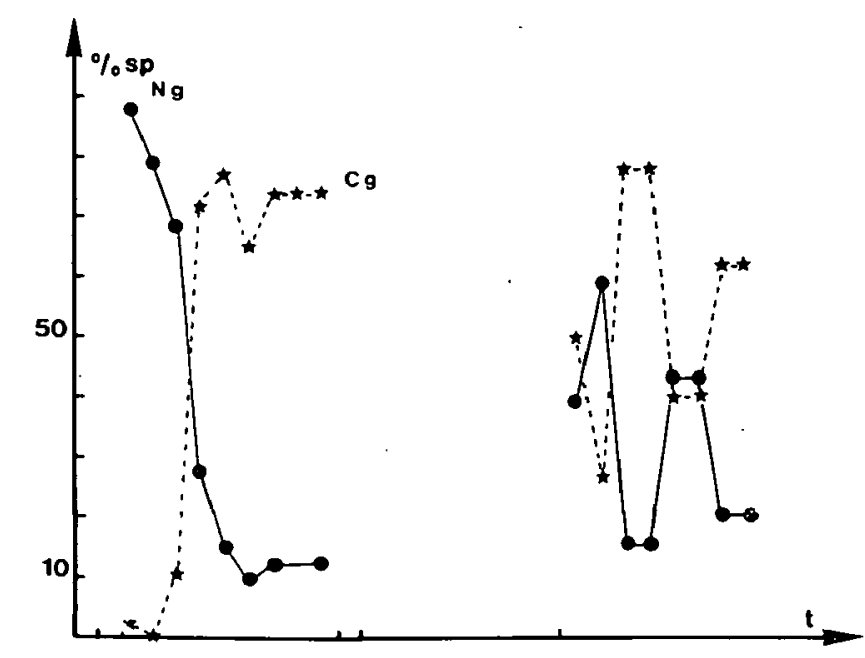

1er pompage: $28 \mathrm{~h}$
(B)
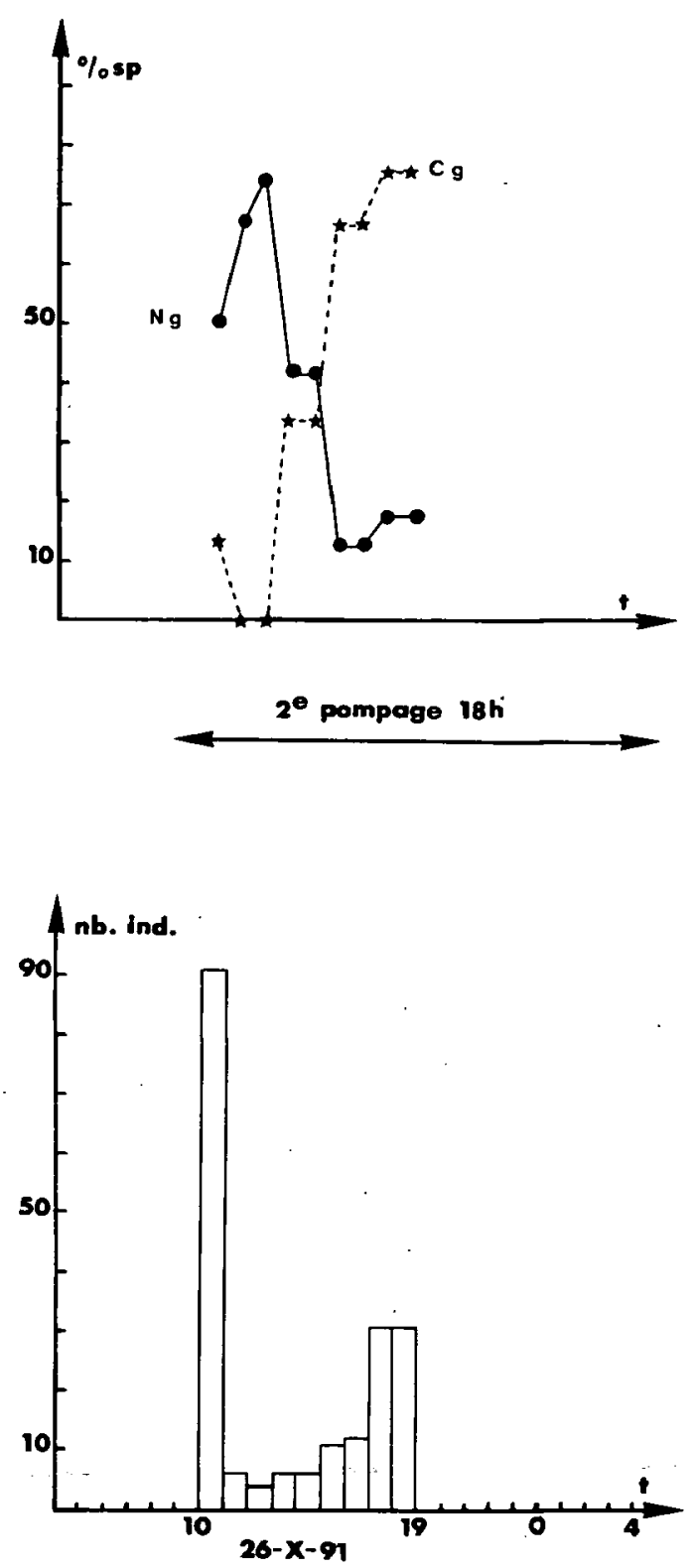

(C)
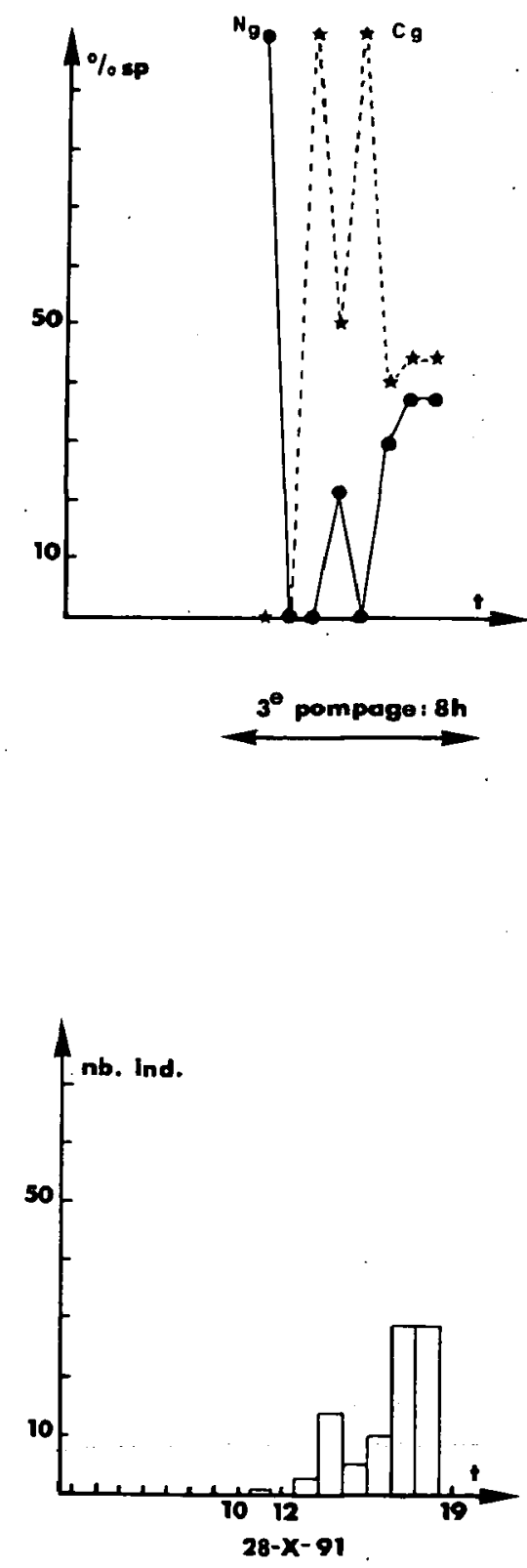

Fig. 4. Pompage dans le gouffre de la Peyrère. Taux de dérive horaire des Harpacticides et évolution de l'abondance relative de Nitocrella gracilis et de Ceuthonectes gallicus dans la partie filtrée des eaux d'exhaure ; a : premier essai ; b : deuxième essai ; $c$ : troisième essai.

Fig. 4. Pumping test in the La Peyrère top window. Drift rate of the harpacticoids in the filtered part of the discharge, and changes in the relative abundance of Nitocrella gracilis and Ceuthonectes gallicus; a : first test ; b : second test ; $c$ : third test.

\subsection{Caractéristiques des prélèvements réalisés dans le gouffre}

8 échantillons de sédiments ont été prélevés dans la galerie dénoyée entre $-4 \mathrm{~m}$ et $-21,5 \mathrm{~m}$ lors du rabattement de la surface piézométrique provoquée par le premier pompage. Les animaux collectés sont beaucoup moins abondants que dans la pêche réalisée en 1975 ou dans les prélèvements de dérive (Tableau 3). Ce résultat ne doit pas surprendre : il rend compte des faibles densités qui existent sous terre. La liste taxonomique établie à partir de ces échantillons n'en est pas moins voisine de celles relatives à la pêche de 1975 et à la dérive due au pompage. Chez les Harpacticides, c'est $C$. gallicus $(62,7 \%)$ et non $N$. gracilis $(24,5 \%)$ qui est dominant.

\subsection{Discussion des données obtenues au cours du pompage}

Les pompages ont provoqué une dérive importante de la microfaune de Crustacés et, notamment, 
des Harpacticides. Les taux de dérive horaire globaux de ces derniers ont varié entre 100 et $9000 \mathrm{i} / \mathrm{h}$, pour des densités de dérive comprises entre 0,1 et $12,6 \mathrm{i} / \mathrm{m}^{3}$. Ces valeurs de dérive sont comparables à celles obtenues à Las Hountas au cours du premier jour de crue des 30 crues étudiées entre 1971 et $1982: 1500<\mathrm{D}<12000 \mathrm{i} / \mathrm{h}$ et $0,5<\mathrm{d}<$ $4 \mathrm{i} / \mathrm{m}^{3}$ (Tableau 2). Ainsi, la dérive totale estimée lors du premier pompage (100 $000 \mathrm{i}$ en 28 heures) est de l'ordre de celle d'un premier jour de crue à Las Hountas où la dérive horaire aurait été de $4000 \mathrm{i} / \mathrm{h}$. Il convient toutefois de remarquer que cette dérive a été provoquée pour un débit moyen de $0,221 \mathrm{~m}^{3} / \mathrm{s}$ alors que, à Las Hountas, les débits à l'origine d'une telle dérive sont compris entre 0,6 et $1 \mathrm{~m}^{3} / \mathrm{s}$, soit 3 à 5 fois plus.

Lors des trois pompages successifs, la dérive n'a cessé de décroître indiquant un dépeuplement notable du site de pompage. Ici encore, les résultats sont comparables à ceux observés à Las Hountas où, au cours d'une crue, la dérive décroît rapidement (Rouch 1974).

Au cours des premières heures des deux premiers pompages, la dérive des Harpacticides est caractérisée par la dominance de $N$. gracilis à qui succède C. gallicus. Le fait que l'on ne retrouve pas ces séquences lors du troisième pompage confirme que le peuplement du site a été fortement perburbé. La dominance de $N$. gracilis au début des deux premiers pompages est en accord avec les données fournies par la pêche de 1975 (Tableau 3). Son remplacement par $C$. gallicus aurait pu être interprété comme étant dû à un apport d'un autre secteur et, notamment, celui de La Hillère dont on a vu qu'il est caractérisé par l'abondance de cette espèce. Cependant, les données hydrologiques indiquent que le secteur de La Hillère a toujours été rapidement déconnecté du drainage et donc de la Peyrère. Or, l'inversion de dominance des espèces d'Harpacticides survient au bout de quatre heures environ. C'est le moment où, le plan d'eau du gouffre ayant baissé de 4 mètres, se produit également une inversion des écoulements au niveau du drainage qui s'effectuent désormais vers la Peyrère. Cette concordance des deux événements (remplacement de $N$. gracilis par C. gallicus et inversion des écoulements) incite à penser que, lorsque le drainage s'est orienté vers la Peyrère, des zones riches en $C$. gallicus et jusqu'alors peu affectées par le pompage, ont été soumises à une dérive.
Les récoltes effectuées in situ lors du dénoyage de la galerie, dominées par $C$. gallicus, diffèrent de la pêche de 1975 et de la dérive des premières heures de pompage. Elles sont beaucoup plus proches du signal obtenu après plusieurs heures de pompage. Deux hypothèses peuvent être formulées : ces prélèvements in situ traduisent soit des apports dus à l'inversion des écoulements, soit un peuplement endobenthique différent du peuplement benthique. Bien que la première de ces hypothèses semble la plus plausible, la seconde ne saurait être totalement écartée.

Le 18 septembre 1992, 11 mois après le pompage, une pêche analogue à celle réalisée en octobre 1975 n'a fourni que 171 Crustacés (Tableau 3). Les Syncarides et l'Harpacticide Parastenocaris mangini ont disparu. C. gallicus $(58 \%$ ) reste l'espèce dominante des Harpacticides où $N$. gracilis est également surpassée par $P$. subterranea. Cette pêche semble indiquer que, un an après, le peuplement des microcrustacés du site de pompage n'a pas récupéré son équilibre antérieur tant qualitatif que quantitatif.

\section{Incidence du pompage sur le peuplement des Harpacticides du système}

Si le pompage a eu un impact évident sur les Harpacticides du gouffre de la Peyrère quelle a été son incidence sur le peuplement de ce groupe dans le système ?

Lors d'un cycle hydrologique moyen, dix à vingt crues affectent le système au cours desquelles une dérive importante d'Harpacticides a lieu non seulement à Las Hountas mais aussi aux exutoires de trop-plein. Bien que le système perde ainsi plusieurs millions d'individus chaque année, l'équilibre de son peuplement n'est pas remis en cause (Rouch 1980).

Comme on l'a vu, il existe une analogie certaine entre la dérive provoquée par le pompage et celle d'une crue où n'interviendrait seulement que Las Hountas et non pas l'ensemble exutoire pérenne exutoires de trop-plein. Dans ces conditions, il était peu probable que le pompage ait pu perturber l'ensemble du système. Toutefois, ce pompage s'est traduit, à trois reprises, par l'assèchement de Las Hountas et, vraisemblablement, d'une partie du drainage. C'est pourquoi, au cours du cycle 1991-1992, une série de crues ont été étudiées à Las Hountas afin de vérifier si la dérive à cet exutoire avait été modifiée tant sur les plans quantitatif que qualitatif. 


\subsection{Les taux de dérive à Las Hountas après le pompage}

Le 5 novembre 1991, une semaine après le pompage, une crue de faible amplitude $\left(n^{\circ} 31\right.$, Tableau 5) est survenue dont le débit maximal a atteint $0,740 \mathrm{~m}^{3} / \mathrm{s}$. Les filtrages réalisés à Las Hountas ce jour-là donnent un taux de dérive moyen de l'ordre de $230 \mathrm{i} / \mathrm{h}$, valeur très inférieure à celles obtenues précédemment (Tableau 2). Afin de confirmer cette observation, deux autres crues à faible débit maximal (la $32=0,615 \mathrm{~m}^{3} / \mathrm{s}$; la $34=0,415 \mathrm{~m}^{3} / \mathrm{s}$ ) ont été étudiées. Elles sont également caractérisées par des dérives horaires moyennes peu élevées puisque leurs valeurs respectives ont été estimées à $582 \mathrm{i} / \mathrm{h}$ et $189 \mathrm{i} / \mathrm{h}$.

Pour expliquer ces valeurs de dérive médiocres, l'hypothèse pourrait être avancée qu'il existe un seuil des débits au-dessous duquel la dérive, qui est de type catastrophique, n'aurait qu'une faible amplitude. Ce pourrait être le cas de la crue 34 (Qmax $=0,415 \mathrm{~m}^{3} / \mathrm{s}$ ). Mais les crues 31 et 32 , par leurs caractéristiques hydrologiques (débit maximal, débit moyen de Las Hountas pendant les filtrages) sont très comparables aux crues 8,22 et 23 (Tableau 2 et Fig. 5). Or, les taux de dérive moyens de ces trois crues ont pour valeur respective 2199,2628 et $1975 \mathrm{i} / \mathrm{h}$, soit 4 à 10 fois plus que lors des crues 31 , 32,34 . Ces données sembleraient donc indiquer que le peuplement du système a été affecté par le pompage.

Deux crues supplémentaires ont été étudiées en 1992. Elles correspondent à des impulsions beaucoup plus fortes avec des débits maximaux respectifs de $4,760 \mathrm{~m}^{3} / \mathrm{s}$ pour la crue 33 et de $10,9 \mathrm{~m}^{3} / \mathrm{s}$ pour la crue 35 . Dans les deux cas, les dérives ont été élevées : $4042 \mathrm{i} / \mathrm{h}$ et $17631 \mathrm{i} / \mathrm{h}$ (Tableau 5). Or, la crue 33 est survenue 8 jours à peine après la crue 32 . Les dérives mises en évidence, au contraire des précédentes, montrent clairement que le peuplement du système n'a pas été perturbé par le pompage puisque leurs valeurs sont conformes (33) voire très supérieures (35) à celles observées antérieurement.

Pour rendre compte de ces résultats contradictoires l'hypothèse suivante peut être formulée. L'assèchement à trois reprises de Las Hountas et, vraisemblablement, d'une partie du drainage, a perturbé le peuplement du drain vers l'aval. Lors des crues de faible amplitude où seule la partie en aval du système est sollicitée, la dérive, avec ses valeurs médiocres, traduirait bien cette perturbation locale. $\mathrm{Au}$ contraire, pendant les fortes crues où l'ensemble du système est sollicité, l'information obtenue, beaucoup plus globale, masquerait les effets locaux dus au pompage.

\subsection{La structure de la dérive}

Chaque exutoire possède sa propre signature fondée sur l'abondance relative des quatre espèces hypogées dominantes dans la dérive (cf. ante). A Las Hountas, sur l'ensemble des 30 premiers jours de crue étudiés entre 1971 et $1982, P$. subterranea, $N$. gracilis, $E$. coiffaiti et $C$. gallicus représentent 99,1\% des 12935 Harpacticides hypogés recueillis. L'abondance relative moyenne de chacune de ces espèces est, respectivement, égale à $65,4 \%-2,2 \%$ $-11.2 \%$ et $20,3 \%$.

Tableau 5. Valeurs moyennes de la dérive des Harpacticides à Las Hountas, le premier jour de crue, lors de 5 crues du cycle 1991-1992 (mêmes légendes que pour le tableau 2).

Table 5. Average values of the harpacticoid drift at the Las Hountas spring during the first day of five floods from the 1991-1992 cycle (same legends as table 2).

\begin{tabular}{lcccccc}
\hline \multicolumn{2}{l}{ Crues } & $\begin{array}{c}\text { Durée } \\
\text { filtr } \\
\text { (h) }\end{array}$ & $\begin{array}{c}\text { QB1 } \\
\text { max } \\
\left(\mathrm{m}^{3}\right)\end{array}$ & $\begin{array}{c}\text { Q Hountas } \\
(\text { filtrage }) \\
\left(\mathrm{m}^{3}\right)\end{array}$ & $\mathrm{D} / \mathrm{h}$ & $\mathrm{d} / \mathrm{m}^{3}$ \\
\hline 31 & 5.11 .91 & 8 & 0,740 & 0,562 & 229 & 0,11 \\
32 & 2.06 .92 & 8 & 0,615 & 0,490 & 582 & 0,32 \\
33 & 10.06 .92 & 8 & 4,760 & 1,168 & 4042 & 0,96 \\
34 & 9.09 .92 & 7 & 0,415 & 0,370 & 189 & 0,14 \\
35 & 5.10 .92 & 7 & 10,900 & 2,170 & 17631 & 2,25 \\
\hline
\end{tabular}




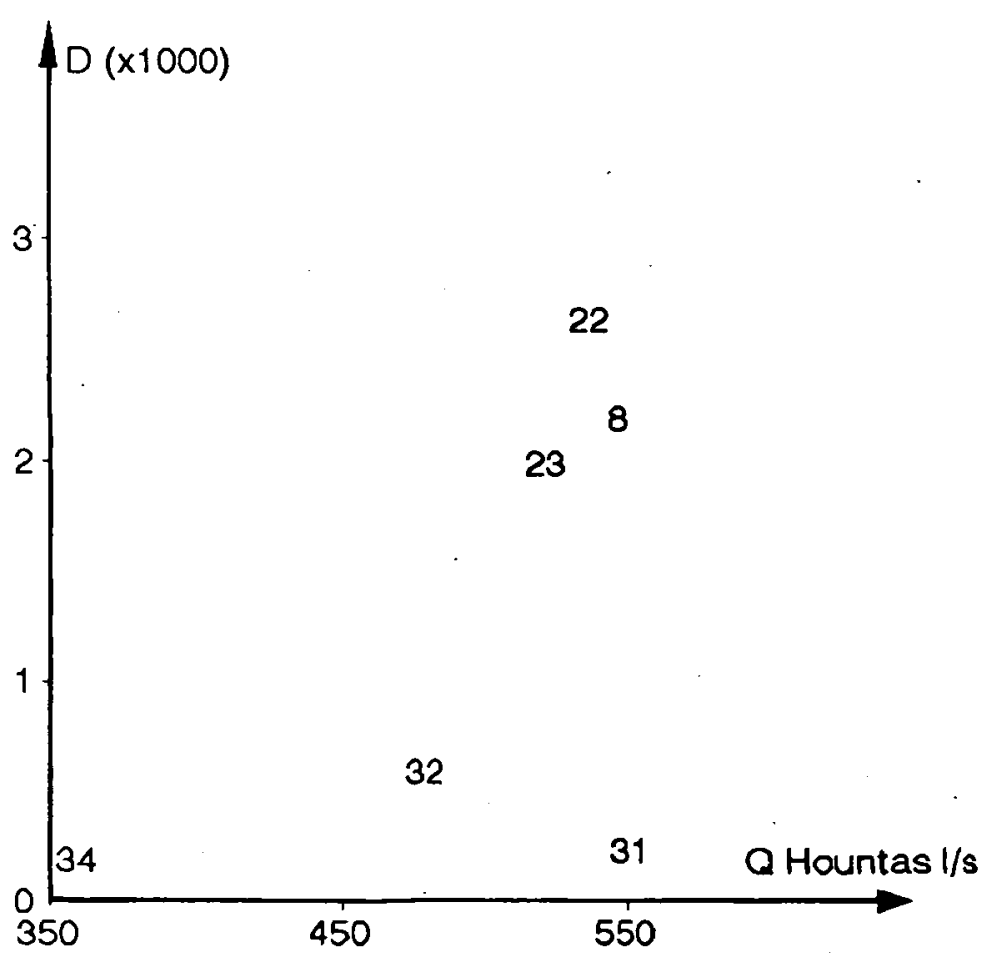

(A)

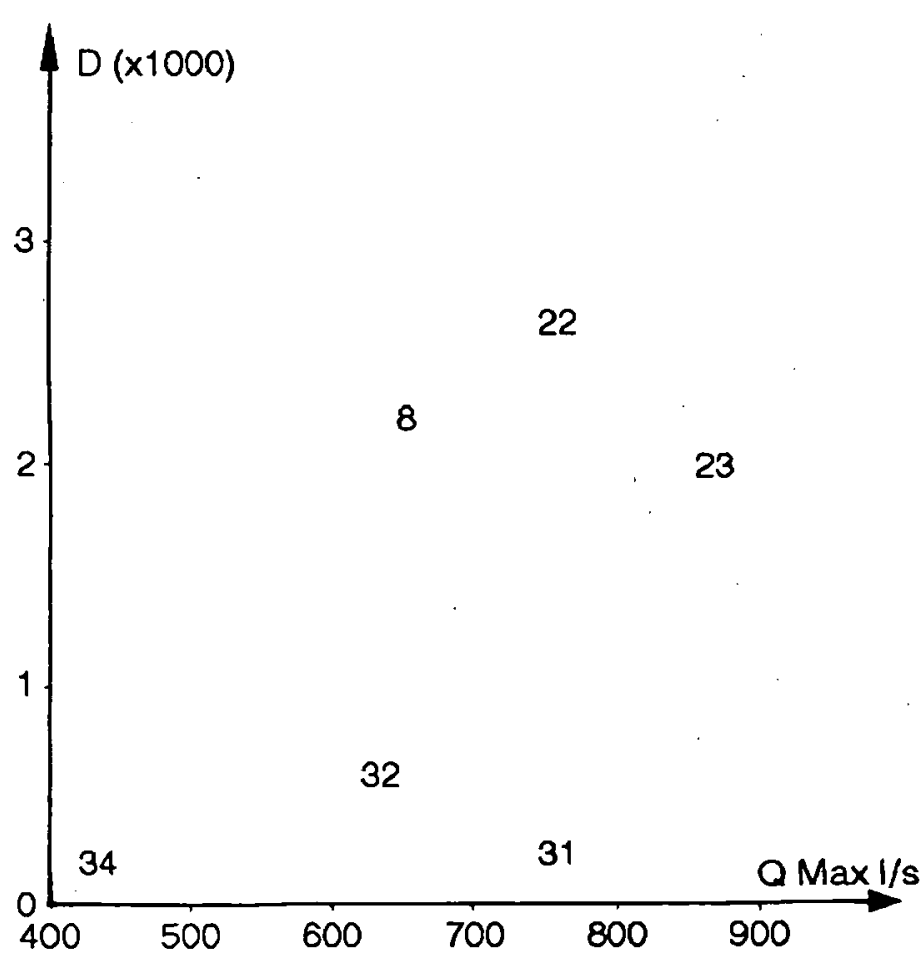

(B)

Fig. 5. Relations entre les taux moyens de dérive (D) et : a) le débit moyen de Las Hountas pendant la période de filtrage, b) le débit maximum du système du Baget, lors des crues $8,22,23,31,32,34$ (voir tableaux 2 et 5 ).

Fig. 5. Relationships between the average drift rates (D) and : a) the mean discharge at the Las Hountas spring during the sampling period, b) the maximum discharge of the Baget karstic system during floods 8, 22, 23, 31, 32, 34 (look at Table 2 and 5).

Afin de voir si le pompage a modifié la structure de ce signal, ces trente crues ont été comparées aux cinq crues étudiées après le pompage par une analyse factorielle des correspondances. Le tableau des données comprenait 4 variables (les quatre espèces hypogées dominantes de la dérive) et les 30 échantillons de dérive (numérotés de 1 à 30 ) récoltés entre 1971 et 1982. 9 échantillons placés en individus supplémentaires, ont été ajoutés (Tableau 6). Cinq d'entre eux $(31,32,33,34,35)$ se rapportent aux crues de Las Hountas postérieures au pompage, les quatre autres aux prélèvements de la Peyrère : la pêche de 1975 (36), la dérive provoquée par le pompage (31), les prélèvements réalisés dans le gouffre (38) et la pêche de 1992 (39). En opérant ainsi, les échantillons supplémentaires n'influent pas sur la structure qui sera uniquement définie à partir de la dérive à Las Hountas antérieure au pompage.

Les deux premiers axes rendent compte de 87,7\% de la variance totale. L'axe 1 est déterminé par $P$. subterranea dans sa partie positive et $C$. gallicus dans sa partie négative. La partie négative de l'axe 2 est caractérisée par $E$. coiffaitii. $N$. gracilis, jamais abondante, n'apparaît que sur l'axe 3.

Dans le plan F1-F2 (Fig. 6), les 30 échantillons relatifs aux crues de Las Hountas entre 1971 et 1982 sont situés à proximité du centre de gravité, ce qui confirme l'homogénéité globale du signal fourni par les espèces hypogées dominantes à cet exutoire.

L'échantillon de dérive 31 , récolté à Las Hountas huit jours après le pompage, s'écarte très nettement du signal habituel en raison de son pourcentage élevé en $C$. gallicus. On assiste ensuite, avec les crues 32 et 33 , à un retour progressif vers une image normale, la crue 33 réintégrant la périphérie du groupe des crues 1 à 30 . Les crues 34 et 35 indiquent un nouvel écart par rapport à la structure type mais de plus faible amplitude. Ce sont les crues à débit peu élevé et à dérive médiocre $(31,32,34)$, donc celles où l'aval du système est surtout sollicité qui s'écartent le plus de la signature habituelle de Las Hountas. Les crues à fort débit et dérive importante . (33 et 35) fournissent une image plus conforme à la normale. 
Tableau 6. Liste des individus de Paraspseudoleptomesochra subterranea, Nitocrella gracilis, Elaphoidella coiffaiti et Ceuthonectes gallicus capturés à Las Hountas (crues 31 à 35) et dans le gouffre de la Peyrère (pêche de $1975: 36$; dérive due au pompage : 37 ; récoltes dans les sédiments : 38 ; pêche de septembre 1992 : 39).

Table 6. Numbers of Parapseudoleptomesochra subterranea, Nitocrella gracilis, Elaphoidella coiffaiti and Ceuthonectes gallicus collected at the Las Hountas spring (floods 31 to 35 ) and in the La Peyrère top window (1975 October sample : 36 ; pumping test drift : 37 ; samples from the sediments : $38 ; 1992$ September sample : 39).

\begin{tabular}{crrrrr}
\hline DATE & $N^{\circ}$ & P.s & N.g & E.c & C.g \\
\hline 5.11 .91 & 31 & 2 & 5 & 4 & 15 \\
2.06 .92 & 32 & 28 & 3 & 11 & 26 \\
10.06 .92 & 33 & 124 & 9 & 31 & 64 \\
9.09 .92 & 34 & 6 & 1 & 2 & 8 \\
5.10 .92 & 35 & 316 & 36 & 67 & 264 \\
2.10 .75 & 36 & 46 & 1107 & 9 & 139 \\
24.28 .10 .91 & 37 & 135 & 290 & 4 & 502 \\
25.10 .91 & 38 & 18 & 46 & & 118 \\
18.09 .92 & 39 & 26 & 2 & & 80 \\
\hline
\end{tabular}

Fig. 6. Analyse factorielle des correspondances sur les échantillons de dérive provenant de Las Hountas (crues 1 à 35) et les prélèvements issus du gouffre de la Peyrère (36 à 39). Seuls les quatre Harpacticides stygobies dominants ont été pris en compte.

Fig. 6. Correspondence analysis on the drift samples from Las Hountas (floods 1 to 35) and the samples from the La Peyrere top window (36 to 39). Only the four dominant stygobiont harpacticoid species have been used.

Peyrère : peche 1992

38

Peyrère : prélèvements 1991

37 Peyrère : pompage 1991

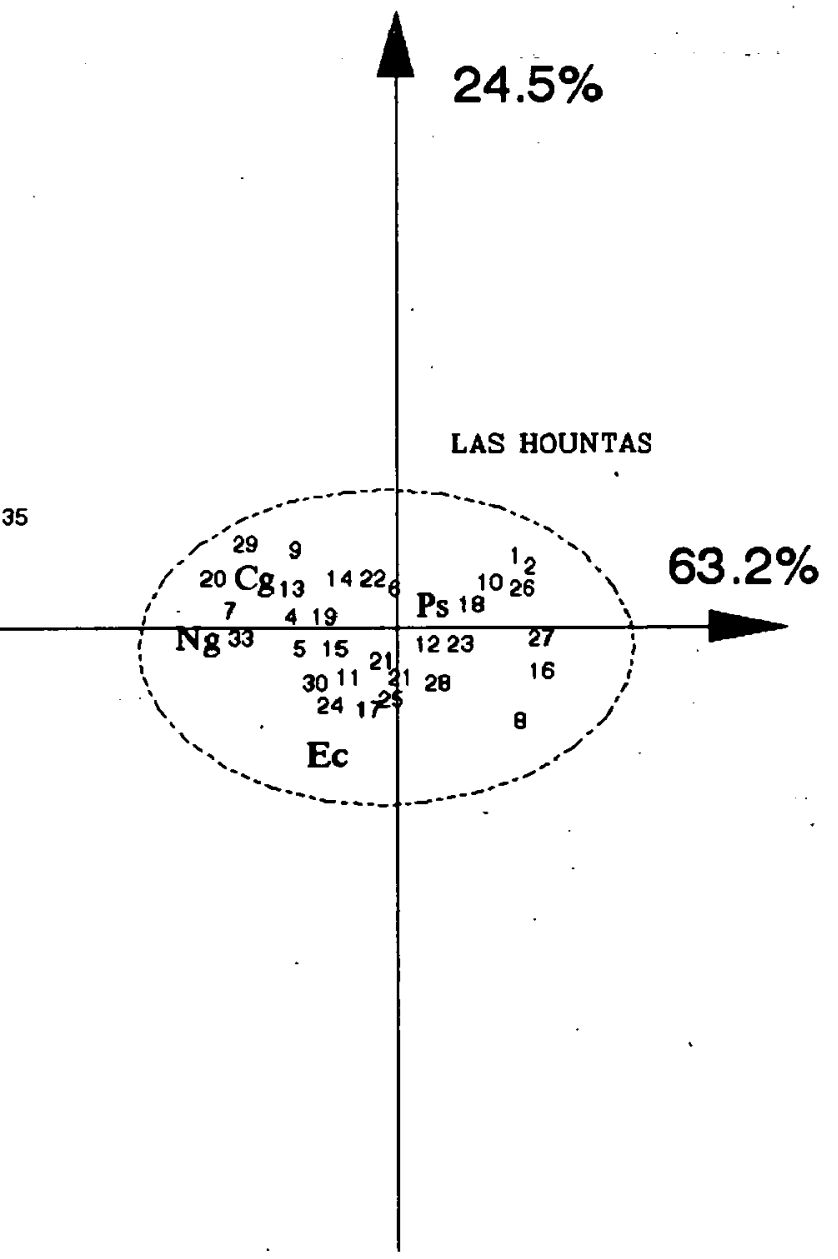


Les prélèvements de la Peyrère, bien qu'assez mal représentés dans le plan F1-F2 n'en traduisent pas moins un certain nombre de faits. Les échantillons 37 et 38 (la dérive provoquée par le pompage et les récoltes in situ), voisins l'un de l'autre, sont également proches du signal fourni par Las Hountas 8 jours après le pompage (31). Les pêches réalisées dans le gouffre en 1975 et 1992 encadrent ces trois échantillons.

On sait que, au cours de la dérive provoquée par le pompage, la structure du signal a changé avec, tout d'abord, la dominance de $N$. gracilis, puis celle de $C$. gallicus. Cet apport en $C$. gallicus marque non seulement le système annexe de la Peyrère (récoltes in situ 38), mais il va influencer également le drainage puisque, huit jours après cette expérience, l'information fournie par Las Hountas est proche de l'image globale donnée par le pompage. La pêche réalisée en septembre 1992 dans le gouffre de la Peyrère (39) montre enfin que, un an après, la structure du peuplement des Harpacticides dans le gouffrè n'est pas revenue à son état antérieur (36) et qu'elle traduit encore l'effet du pompage.

Ainsi, le pompage a eu un effet local sur le peuplement du drainage vers l'aval ; la structure de celui-ci, profondément modifiée tout d'abord, a tendu à revenir vers la normale au cours du cycle hydrologique 91-92.

\section{Conclusions}

Le pompage à gros débit réalisé dans le gouffre de la Peyrère, regard sur le karst noyé du système karstique du Baget, a provoqué une dérive de la microfaune des Crustacés aquatiques stygobies de ce secteur. Celle des Harpacticides, dont les valeurs sont comprises entre 100 et $9000 \mathrm{i} / \mathrm{h}$, est relativement importante et comparable à la dérive observée à l'exutoire pérenne du système, Las Hountas, le premier jour d'une crue.

Cette dérive des Harpacticides a beaucoup diminué au cours des trois essais successifs de l'expérience traduisant un dépeuplement du secteur où s'effectuait le pompage. Ce résultat est conforté par le fait que le signal de sortie, bien structuré lors des deux premiers essais, s'est fortement altéré lors du troisième. Un an plus tard, il semblerait que le peuplement des Harpacticides dans le gouffre de la Peyrère n'ait pas retrouvé sa structure antérieure au pompage.
Ce pompage a également affecté le peuplement du drainage en aval du système. En effet, au cours du cycle hydrologique qui a suivi cette expérience, la dérive des Harpacticides relative à cette partie du drainage a diminué. Toutefois, le peuplement global des Harpacticides du système n'a pas été remis en cause. En effet, lors des fortes crues qui ont sollicité l'ensemble du bassin versant, les taux de dérive ont présenté des valeurs identiques à ce qu'elles étaient avant cette expérience.

La structure de la dérive des Harpacticides hypogés a été également perturbée, notamment juste après le pompage. Cependant, au fil des crues étudiées, cette structure a montré une nette tendance au retour vers la normale sans l'atteindre complètement.

Que les données biologiques fournies par cette expérience n'aient pas pu être interprétées sans les connaissances préalables accumulées sur le système du Baget prouve, une fois encore, le bien-fondé de l'approche des aquifères karstiques à l'échelle du système-bassin versant (Mangin 1974, 1975, Rouch 1977, Bakalowicz 1979, Turquin 1981, Gibert 1986). C'est à ce niveau d'échelle qu'apparaissent les interactions entre le peuplement, d'une part, la structure et le fonctionnement de l'aquifère, d'autre part. De ce point de vue, la sensibilité du peuplement des Harpacticides à l'impact du pompage réalisé sur le système du Baget paraît exemplaire. Ce résultat ne peut qu'inciter à prendre davantage en compte la composante biologique des aquifères lorsque ceuxci font l'objet d'expérimentation ou d'exploitation.

\section{Remerciements}

Nous remercions A. Mangin, M. Bakalowicz, D. D'Hulst, L. Danneville, B. Marsaud du Laboratoire souterrain, J. Ricard et G. Chevalier du BRGM, G. Jauzion de la SMPS ainsi que tous les membres de l'entreprise Hydrokarst pour l'aide qu'ils nous ont apportée sur le terrain. A. Mangin, M. Bakalowicz par leurs commentaires avisés, D. D'Hulst pour la partie informatique, nous ont été d'un précieux secours. Les figures définitives de cette note sont dues à Mme Cazals. F. Boineau a saisi le manuscrit.

\section{Travaux cités}

Andrieux C. 1972. - Le système karstique du Baget (Ariège). 1. Sur la thermique des eaux au niveau de l'exutoire principal (note préliminaire). Ann. Spéléol., 27 : 525-541.

Bakalowicż M. 1979. - Contribution de lă géochimie des eaux à la connaissance de l'aquifère karstique et de la karstification. Thèse Univ. Paris 6, 269 p. 
Gibert J. 1986. - Ecologie d'un système karstique jurassien ; hydrogéologie, dérive animale, transits de matières, dynamique de la population de Niphargus (Crustacés Amphipodes). Mém. Biospéol., $13: 330$ p.

Mangin A. 1974, 1975. - Contribution à l'étude hydrodynamique des aquifères karstiques. Ann. Spéléol., 29, $3: 283-332$; $29,4: 495-601 ; 30,1: 21-124$.

Rouch R. 1970. - Le système karstique du Baget. I. Le phénomène d' " hémorragie " au niveau de l'exutoire principal. Ann. Speléol., 25 : 665-709.

Rouch R. 1972. - Le système karstique du Baget. II. Etude des Harpacticides rejetés au niveau de Las Hountas au cours de plusieurs crues du cycle hydrologique 1970-1971. Ann. Spéléol., 27 : 139-176.

Rouch R. 1974. - Le système karstique du Baget. III. Etude des sorties d'Harpacticides au niveau de Las Hountas lors de plusieurs crues des cycles hydrologiques 1971-1972 et 1972-1973. Ann. Spéléol., 29 : 351-372.

Rouch R. 1977. - Considérations sur l'écosystème karstique. C.R. Acad. Sc. Paris, 284 : 1101-1103.
Rouch R. 1980. - Le système karstique du Baget. X. La communauté des Harpacticides. Richesse spécifique, diversité et structures d'abondances de la nomocenose hypogée. Annls Limnol., $16: 1-20$.

Rouch R. 1982. - Le système karstique du Baget. XIII. Comparaison de la dérive des Harpacticides à l'entrée et à la sortie de l'aquifère. Annls Limnol., 18 : 133-150.

Rouch R. \& Bonnet L. 1976. - Le système karstique du Baget. IV. Premières données sur la structure et l'organisation de la communauté des Harpacticides. Ann. Spéléol., 31 : 27-41.

Rouch R. \&!Bonnet L. 1977. - Le système karstique du Baget. VII. La communauté des Harpacticides. Structure du peuplement hypogé pendant les crues. Annls Limnol., 13 : 251-259.

Turquin M.J. 1981. - Profil démographique et environnement chez une population de Niphargus virei (Amphipode troglobie). Bull. Soc. Zool. Fr., 106 : 457-466. 